\title{
The pursuit of pleasure
}

\section{Chronicles of reward processing discoveries and emerging roles for big data analytics in ma- jor depression disorder treatment}

\author{
Roger Hudson, Nirushan Puvanenthirarajah
}

\section{ABSTRACT}

Many component processes of reward require appropriate serotonin (5HT) and dopamine (DA) neurotransmission within key limbic brain regions. Evidence suggests that dysregulation of 5HT and DA transmission can precipitate reward dysfunction and major depressive disorder (MDD) symptoms in genetically predisposed individuals. Various neurobiological indicators (biomarkers) of MDD have been proposed, including changes in signal transduction pathways, protein phosphorylation, and gene expression in subcortical, reward-related structures. However, these insights have yielded limited clinically relevant benefits for diagnosis, treatment, or prognosis. In addition, clinical application of identified biomarkers is often hindered by multiple factors including disease heterogeneity and symptom variability between patients. Innovative approaches including big data analytics, methodical collaboration between research programs, and reverse-translational strategies are now required to understand whether particular biomarkers can be used to predict disease onset and treatment response, to stratify treatments for patient subgroups, to and develop novel pharmacotherapies. This review briefly summarizes the predictive value of big data analytics in parsing the neurobiological underpinnings of MDD, with a focus on potential clinically viable biomarkers for predictive therapies.

\section{INTRODUCTION}

Major depressive disorder (MDD) is a debilitating mood disorder characterized by blunted reward functioning., ${ }^{1,2}$ Investigations into the neurobiology of reward processing suggest that dysregulated neurotransmission and molecular signaling cascades within principal reward-related brain regions are critically involved in MDD pathophysiology..$^{2-4}$ In fact, many MDD pharmacotherapies act to normalize activity within these brainreward pathways. ${ }^{5}$ It is conceptualized that candidate biological indicators (biomarkers) of MDD pathophysiology and treatment outcome may be used by physicians to guide diagnosis and predict optimal individualized therapies. Importantly, establishing precise biomarker-derived diagnostic tests would allow physicians to treat the illness with more pharmacological precision, as receptor profiles and pharmacokinetics vary widely between drugs. Largescale efforts to detect clinically-relevant biomarkers using common measures (eg blood tests) are currently underway, ${ }^{6,7}$ but the quantity of biological and genetic data generated hinders efficiency in analysis. ${ }^{8}$ As such, there has been a great increase in the use of big data analytics, involving the collection of tremendously large datasets that can be analyzed to reveal patterns, trends, and associations. ${ }^{78}$ This analytic framework may serve to bridge the gap between the current status of MDD treatment and individually optimized therapeutic regimens..$^{6-8}$

Insights into the neurobiology of reward reveal that dopamine (DA) and serotonin (5HT) neurotransmission each facilitate reward functioning in the mammalian brain. ${ }^{910}$ For example, depletion of the DA or $5 \mathrm{HT}$ precursors tyrosine or tryptophan reduces subjective pleasure ratings in unmedicated patients with MDD, but not in their medicated counterparts. ${ }^{10,11}$ This is consistent with evidence from preclinical animal models demonstrating that enhanced DA and 5HT transmission reduces behavioural and psychomotor impairments associated with MDD symptoms (see Table 1 for description of MDD symptoms)..$^{12,13}$ Despite the emergence of selective 5HT reuptake inhibitors (SSRIs) and other agents, MDD treatment responses remain less than optimal. In fact, only $30 \%$ of patients achieve complete symptom resolution with antidepressant therapy ${ }^{14,15}$ and those with residual symptoms are predisposed to relapse. ${ }^{16}$ Thus, big data analytics together with extensive biomarker datasets may represent a potential methodology to obtain a greater understanding of the underlying processes that regulate patient responses to treatment.

Immense datasets generated by high-throughput genetic, molecular, and neuroimaging techniques increasingly rely on big data analytics for efficient sorting, normalization and processing. ${ }^{7}$ Challenges with transcription and analysis of large datasets are typically categorized as those of volume (size of dataset), speed (rapid acquisition), or diversity (from multiple sources). ${ }^{17}$ Big data analytics often employ machin -learning techniques to locate patterns within and between datasets that mainstream technology and human throughput cannot efficiently detect. ${ }^{8}$ These analysis methods can be utilized to parse vast datasets of biological or genetic samples, complex data derived from imaging studies, and social media information, among others. A shift toward electronic formats has increased access to clinical data, including physician notes, which may also serve as potential big data sources. ${ }^{17}$

Due to variation in MDD treatment outcomes, categorizing aspects of genetic profiles with treatment success versus failure may aid clinicians in predicting patient responses to medications. ${ }^{718}$ Developing strategies for individualized therapies is important because measures such as drug substitution or addition are only initiated following a medication trial of sufficient duration, which could be 2 months or longer. ${ }^{19}$ However, half of all patients without symptom improvement cease treatment within this time. ${ }^{20}$ 
Therefore, prompt diagnosis and definitive treatment of MDD are crucial to reduce prospects of future MDD episodes. This review briefly summarizes the predictive potential of big data techniques in parsing the neurobiological underpinnings of MDD, with a focus on potential clinically viable biomarkers for predictive therapies.

Table 1. Diagnostic and Statistical Manual of Mental Disorders (DSM-5) Criteria for Major Depressive Disorder ${ }^{a}$

\begin{tabular}{|c|c|}
\hline Symptoms ${ }^{b}$ & Description \\
\hline 1 & Depressed mood most of the day \\
\hline 2 & Diminished interest or pleasure in all or most activities typically enjoyed \\
\hline 3 & Significant unintentional weight loss or gain \\
\hline 4 & Insomnia or sleeping too much \\
\hline 5 & Agitation or psychomotor retardation noticed by others \\
\hline 6 & Fatigue or loss of energy \\
\hline 7 & Feelings of worthlessness or excessive guilt \\
\hline 8 & Diminished concentration abilities or indecisiveness \\
\hline 9 & Recurrent thoughts of death \\
\hline $\begin{array}{l}\text { at leas } \\
\text { 2) depre } \\
{ }^{b} \text { Confirm } \\
\text { clinically } \\
\text { importan } \\
\text { Abbrevia } \\
\text { edition; } 1\end{array}$ & $\begin{array}{l}\text { tone symptom must be 1) anhedonia (diminished interest or pleasure), or } \\
\text { issed mood. } \\
\text { nation of diagnosis requires presence of at least } 5 \text { symptoms that cause } \\
\text { significant distress or impairment in social, occupational, or other } \\
\text { t areas of functioning. } \\
\text { ations: DSM-5, Diagnostic and Statistical Manual of Mental Disorders, fifth } \\
\text { MDD, Major Depressive Disorder }\end{array}$ \\
\hline
\end{tabular}

\section{CHRONICLING THE ROLES OF SEROTONIN AND DOPAMINE IN REWARD PROCESSING}

Reward processing deficits are among the chief symptoms of MDD, and may involve anomalies in multiple component processes including psychomotor and motivational domains. ${ }^{27,28}$ Motivation for rewarding experiences, as well as the ability to select appropriate behaviours based on anticipated reward, are fundamental to the normal processing of hedonic stimuli. ${ }^{29}$ These capabilities may be even more important in disorders such as MDD, in which typical hedonic capacity and motivation to seek out and respond to rewarding stimuli are disturbed. ${ }^{9,30}$ As stated earlier, the neurotransmitters DA and 5HT each act to facilitate appropriate reward function. ${ }^{9,10}$ Motivational and memory components of reward are crucially mediated by DA transmission in limbic regions including the nucleus accumbens (NAcc) and hippocampus. ${ }^{21,22}$ In contrast, $5 \mathrm{HT}$ influx to the hypothalamus and cortical regions modulates mood, stress, and emotional valence, ${ }^{11,23,24}$ and $5 \mathrm{HT}$ transmission to striatal regions moderates DA-driven actions on reward function. ${ }^{25}$

Early evidence from experimental animals suggested that DA is critically involved in reward processing. Seminal research into intracranial self-stimulation (ICSS) revealed that subjects learned to press a lever to receive reinforcing currents of brain stimulation..$^{31}$ The electrical stimulation was delivered to the medial forebrain bundle, a collection of nerve fibers partly originating in the ventral tegmental area (VTA) of the midbrain. ${ }^{32,33}$ Fluorescence histochemistry evidence demonstrated that maximal ICSS response rates were maintained when delivered to the VTA and substantia nigra, the 2 principal regions containing DA cell bodies. ${ }^{34-36}$ Furthermore, experiments using 6-hydroxydopamine ablation and microdialysis techniques further demonstrated that the NAcc was explicitly activated following VTA ICSS, ${ }^{37}$ and that blockade of DA release in the NAcc considerably reduces motivated responding. ${ }^{38}$ Current indications suggest that this particular DA-driven circuit is essential for motivated behaviour in the context of affective stimulus processing. ${ }^{38,39}$

Beyond the role of $5 \mathrm{HT}$ in mood regulation and stress, signaling at $5 \mathrm{HT}$ receptors can temper DA neurotransmission and its associated actions on reward processing. For example, 5HT and DA neurotransmission interact to influence striatal reward responses and their conditioned cues depending on the predicted reward value. ${ }^{24,39}$ Downstream signal transduction substrates associated with 5 HT receptor activation have also been linked to the actions of these neurochemicals on reward processing, particularly in MDD. ${ }^{40}$ For example, successful treatment responses in patients undergoing pharmacotherapy for MDD are marked by increased expression of hippocampal brain-derived neurotrophic factor (BDNF). ${ }^{5,6}$ Variations in BDNF expression following treatment with SSRIs and other antidepressants are also related to changes in growth factor expression, ${ }^{41}$ neuroendocrine and thyroid function, ${ }^{42}$ neuroinflammation, ${ }^{43}$ and measures of $5 \mathrm{HT}$ and DA metabolites. ${ }^{44}$

Although putative biomarkers have been identified, their functions are largely undefined. Thus, prospects of predicted diagnosis and treatment response have not yet been realized. This burden is attributable to the heterogeneity of MDD, methodological variation in research, and the large variety of biomarkers, in which expression often varies according to several factors. ${ }^{6}$ To address this issue, big data analytics and machine learning techniques are being applied to parse alleged MDD biomarkers. ${ }^{7,8}$

\section{ROLES FOR BIG DATA ANALYTICS IN THE TREATMENT OF MAJOR DEPRESSIVE DISORDER}

Recent large-scale research initiatives characterizing MDD biomarkers have integrated functional magnetic resonance imaging data with electroencephalography and genetic, proteomic, and genomic profiling., ${ }^{718}$ These platforms assess large cohorts of medicated patients, unmedicated patients, and control subjects to deconstruct MDD phenotypes through use of machine learning and multivariate techniques. ${ }^{45}$ Researchers and clinicians seek to quantify predictors, moderators, and mediators of MDD onset and treatment response that are measurable through common clinical assays. As such, a primary goal of big data analytics in this context is to inform individualized approaches to MDD treatment through identification of clinical biomarkers associated with treatment response. ${ }^{7,18,45}$

Following the parsing of thousands of candidate biomarkers from their associated pharmacotherapeutic correlates, preclinical animal models can further target biomarkers involved in treatment 
efficacy to inform individualized MDD treatment and drug development. Indeed, big data tools can be useful for generating hypotheses, but these hypotheses must be rigorously tested using clinical and preclinical research tools to integrate insights into clinical practice. ${ }^{28,47}$ Thus, methodologies from mid-late 20 th century research on reward systems will unquestionably continue to inform research throughout the era of big data analytics. Furthermore, the applications of big data analyses are not limited to detecting druggable biomarkers. They may also be used to distinguish MDD from other symptomatically similar neuropsychiatric illnesses (i.e. schizophrenia, bipolar disorder, catatonia) or other depressive disorders (i.e. persistent depressive disorder, reactive depression), and to further characterize differences within MDD subpopulations that require more specific drug therapy. Lastly, these techniques can also provide insights into the neurobiology underlying treatment responses to nonpharmacological, evidence-based interventions for MDD including transcranial magnetic stimulation, cognitive behavioural therapy, cognitive remediation, and others., ${ }^{7,4}$

Despite its benefits, scientific and technological challenges arise from this 'big data' predicament. ${ }^{46}$ For example, preclinical studies assessing the viability of biomarkers are typically limited in sample size, and often vary in experimental methodology. ${ }^{6,7}$ Furthermore, appropriate translation of novel, preclinicallyidentified biomarkers to contexts of MDD will require extensive replication in double-blind placebo-controlled studies. These studies require exceptionally large cohorts to establish results, implications in heterogeneous patient subpopulations, and any correlation or divergence of biomarkers according to pharmacotherapy regimen. Thus, large-scale collaborative efforts are needed to ensure standardized methodology, measurement, and data normalization across laboratories. This will ultimately guide psychiatry toward translational, applied research in MDD.,8

\section{CONCLUSIONS}

Disturbances in 5HT and DA neurotransmission have been implicated in reward dysfunctions underlying MDD. Although changes in downstream signal transduction pathways and gene expression have been linked to MDD, these data have not been overtly successful in enhancing diagnosis, treatment, or prognosis. Many factors contribute to this disconnection, including disease heterogeneity and biomarker variability. Innovative approaches including big data analytics, systematic collaboration between research programs, and reverse-translational strategies are now necessary to understand whether biomarkers are useful targets for predicting disease onset and treatment response, to stratify treatments for patient subgroups, and to develop novel pharmacotherapies. By expanding on these protocols, there is hope for improving the lives of many individuals with MDD.

\section{REFERENCES}

1. Kumar P, Waiter G, Ahearn T, et al. Abnormal temporal difference reward-learning signals in major depression. Brain. 2008;131(8):208493. https://doi.org/10.1093/brain/awn136

2. Whitton AE, Treadway MT, Pizzagalli DA. Reward processing dysfunction in major depression, bipolar disorder and schizophrenia. Curr Opin Psychiatry. 2015;28(1):7. https://doi.org/10.1097/ YCO.0000000000000122

3. Schultz W. Multiple reward signals in the brain. Nat Rev Neurosci. $2000 \mathrm{Jan} ; 1(3): 199$

4. Heyman E, Gamelin FX, Goekint M, et al. Intense exercise increases circulating endocannabinoid and BDNF levels in humans-Possible implications for reward and depression. Psychoneuroendocrinology. 2012;37(6):844-51.

5. Lee BH, Kim YK. The roles of BDNF in the pathophysiology of major depression and in antidepressant treatment. Psychiat Invest. 2010;7(4):231-5.

6. Strawbridge R, Young AH, Cleare AJ. Biomarkers for depression: recent insights, current challenges and future prospects. Neuropsychiatr Dis Treat. 2017;13:1245-62. https://doi.org/10.2147/ NDT.S114542

7. Lam RW, Milev R, Rotzinger S, et al. Discovering biomarkers for antidepressant response: protocol from the Canadian biomarker integration network in depression (CAN-BIND) and clinical characteristics of the first patient cohort. BMC Psychiatry. 2016;16(1):105. http://doi.org/10.1186/s12888-016-0785-x

8. Boksa P. A way forward for research on biomarkers for psychiatric disorders. J Psychiatry Neurosci. 2013 Jan;38(2):75-7.

9. Schultz W. Predictive reward signal of dopamine neurons. J Neurophys. 1998;80(1):1-27.

10. Ruhé HG, Mason NS, Schene AH. Mood is indirectly related to serotonin, norepinephrine and dopamine levels in humans: a metaanalysis of monoamine depletion studies. Mol Psych. 2007;12(4): 331-59.

11. Rogers RD, Tunbridge EM, Bhagwagar Z, et al. Tryptophan depletion alters the decision-making of healthy volunteers through altered processing of reward cues. Neuropsychopharm. 2003;28(1): 153.

12. Der-Avakian A, Markou A. The neurobiology of anhedonia and other reward-related deficits. Trends Neurosci. 2012;35(1):68-77.

13. Hudson R, Zhou Y, Leri F. The combination of escitalopram and aripiprazole: Investigation of psychomotor effects in rats. J Psychopharm. 2017;31(12):1605-14.

14. Fawcett J. Antidepressants: partial response in chronic depression. $\mathrm{Br}$ J Psychiatry Suppl. 1994;26:37-41.

15. O'Reardon JP, Amsterdam JD. Treatment-resistant depression: progress and limitations. Psychiatr Ann. 1998 Jan;28(11):633-40.

16. Paykel ES, Ramana R, Cooper Z, et al. Residual symptoms after partial remission: an important outcome in depression. Psychol Med. 1995;25:1171-80.

17. Stewart R, Davis K. "Big data" in mental health research: current status and emerging possibilities. Soc Psych Epidemiol. 2016;51:1055-72. doi. org/10.1007/s00127-016-1266-8

18. Kennedy S, Downar J, Evans K, et al. The Canadian biomarker integration network in depression (CAN-BIND): advances in response prediction. Curr Pharm Des. 2012;18(36): 5976-89.

19. Ferguson JM. SSRI antidepressant medications: adverse effects and tolerability. J Clin Psych. 2001;17(3):8-12.

20. Melfi CA, Chawla AJ, Croghan TW, et al. The effects of adherence to antidepressant treatment guidelines on relapse and recurrence of depression. Arch Gen Psychiatry. 1998;55:1128-32.

21. Salamone JD. The involvement of nucleus accumbens dopamine in appetitive and aversive motivation. Beh Brain Res. 1994;61(2):117-33. 
22. Otmakhova N, Duzel E, Deutch AY, Lisman J. The hippocampalVTA loop: the role of novelty and motivation in controlling the entry of information into long-term memory. In Intrinsically motivated learning in natural and artificial systems. In: Baldassarre G, Mirolli M, eds. Intrinsically motivated learning in natural and artificial systems. Berlin: Springer-Verlag; 2013. p. 235-54. https://doi.org/10.1007/978-3642-32375-1_10

23. Kranz GS, Kasper S, Lanzenberger R. Reward and the serotonergic system. J Neurosci. 2010;166(4):1023-35.

24. Zhong W, Li Y, Feng Q, et al. Learning and stress shape the reward response patterns of serotonin neurons. J Neurosci. 2017;37(37): 886375.

25. Daw ND, Kakade S, Dayan P. Opponent interactions between serotonin and dopamine. Neural Netw. 2002;15(4):603-16.

26. Labonté B, Engmann O, Purushothaman I, et al. Sex-specific transcriptional signatures in human depression. Nat Med. 2017;23(9):1102.

27. Berridge KC, Robinson TE. Parsing reward. Trends Neurosci. 2003;26(9):507-13.

28. Hudson R. Parsing anhedonia: a reverse-translational strategy for treatment of anhedonia in clinical populations and potential implications of conditioned motivators. UWOMJ. 2017;86(2):44-7.

29. Redlich R, Dohm K, Grotegerd D, Opel N et al. Reward processing in unipolar and bipolar depression: a functional MRI study. Neuropsychopharm. 2015;40(11): 2623.

30. Pizzagalli DA, Iosifescu D, Hallett LA, et al. Reduced hedonic capacity in major depressive disorder: evidence from a probabilistic reward task. J Psychiatry. 2008;43:76-87.

31. Olds J. Self-stimulation of the brain. Science. 1958;127(3294):315-24.

32. Olds J. Effects of lesions in medial forebrain bundle on self-stimulation behavior. Am J Physiology-Legacy Content. 1969;217(5):1253-64.

33. Clavier RM, Routtenberg A. Brain stem self-stimulation attenuated by lesions of medial forebrain bundle but not by lesions of locus coeruleus or the caudal ventral norepinephrine bundle. Brain Res. 1976;101(2):251-71.

34. Beckstead RM, Domesick VB, Nauta WJ. Efferent connections of the substantia nigra and ventral tegmental area in the rat. Brain Res. 1979;175(2):191-217.

35. Clavier RM, Routtenberg A. Ascending monoamine-containing fiber pathways related to intracranial self-stimulation: histochemical fluorescence study. Brain Res. 1974;72(1):25-40.

36. Corbett D, Wise RA. Intracranial self-stimulation in relation to the ascending dopaminergic systems of the midbrain: a moveable electrode mapping study. Brain Res. 1980;185(1):1-15.

37. Hand TH, Franklin KB. 6-OHDA lesions of the ventral tegmental area block morphine-induced but not amphetamine-induced facilitation of self-stimulation. Brain Res. 1985;328(2):233-41.

38. Garris PA, Kilpatrick M, Bunin MA, Michael D, Walker QD, Wightman RM. Dissociation of dopamine release in the nucleus accumbens from intracranial self-stimulation. Nature. 1999;398(6722):67.

39. Hollerman JR, Schultz W. Dopamine neurons report an error in the temporal prediction of reward during learning. Nature Neurosci. 1998;1(4):304.

40. Grønli J, Bramham C, Murison R, et al. Chronic mild stress inhibits BDNF protein expression and CREB activation in the dentate gyrus but not in the hippocampus proper. Pharm Biochem Beh. 2006;85(4):842-9.

41. Warner-Schmidt JL, Duman RS. VEGF as a potential target for therapeutic intervention in depression. Curr Op Pharmacol. 2008;8(1):14-19.
42. Stetler C, Miller GE. Depression and hypothalamic-pituitary-adrenal activation: a quantitative summary of four decades of research. Psychosom Med. 2011;73(2):114-26.

43. Strawbridge R, Arnone D, Danese A, et al. Inflammation and clinical response to treatment in depression: A meta-analysis. Eur Neuropsychopharmacol. 2015;25(10):1532-

44. Atake K, Yoshimura R, Hori H, et al. Duloxetine, a selective noradrenaline reuptake inhibitor, increased plasma levels of 3-methoxy-4-hydroxyphenylglycol but not homovanillic acid in patients with major depressive disorder. Clin Psychopharmacol Neurosci. 2014;12(1):37-40.

45. Lopez JP, Kos A, Turecki G. Major depression and its treatment: microRNAs as peripheral biomarkers of diagnosis and treatment response. Curr Op Psych. 2018;31(1):7-16.

46. Kessler RC, van Loo HM, Wardenaar KJ, et al. Testing a machinelearning algorithm to predict the persistence and severity of major depressive disorder from baseline self-reports. Mol Psychiatry. 2016;21(10):1366.

47. Lam RW, Kennedy SH. STAR* D and measurement-based care for depression: Don't toss out the baby!. Can J Psychiatry. 2015 Jan; 60(1):6-8. https://doi.org/10.1177/070674371506000103

48. MacQueen G. Neuroimaging and electrophysiology in predicting treatment responsiveness in depression: bridging the lab-to-clinic divide? Can J Psychiatry. 2013;58(9):497. 